\title{
Some Practical Aspects of Hydrogen Electrode Measurements'
}

\author{
By Paul E. Klopsteg ${ }^{2}$
}

Central Schentific Co., Chicago, Illinois

$\mathrm{T}$ $\mathrm{HE}$ electrical (physical) measurements by which $\mathrm{H}$-ion concentration can be determined, and the physicochemical theory upon which the measurements are based, are somewhat off the paths trodden by the average chemist, and for this reason he is likely to make only their casual acquaintance. It is not strange that this should be so; for there are so many new things purely chemical with which the average chemist must keep abreast that a subject which is only indirectly chemical must necessarily be put on the waiting list, where it usually remains. Besides, there have been so many hundreds of papers in the H-ion literature of the past half dozen years that the probability of any particular one of them having the sort of information he wants is small indeed.

If the writer has correctly interpreted the desires he has heard expressed, he would say that what the chemist wants to know about electrometric methods is what they can do for him in the solution of his own problems. Having the assurance that he can utilize the method, he wants to know how to proceed to get his results with least effort and outlay, yet with such ease, directness, and accuracy, that the net balance is a saving of time and money. This paper is an endeavor to answer these questions as accurately as possible; or, where a definite answer cannot be given, to point out the reason, and to suggest the best procedure for finding the answer.

Electrical methods of measurement and control in chemistry are possible because of the essentially electrical nature of the mechanism of a chemical reaction, $i$. e., of the ions involved in the reaction. There are two distinctly different electrical properties of solutions that can be used to produce or control mechanical effects in a manner corresponding to chemical changes in the solution. 'These are, first, conductance, the property of a solution which permits an electric current to pass more or less freely. The second is the characteristic which results in a potential difference or electromotive force being set up between the solution and an appropriate electrode immersed in it. Both properties depend on the ion, or the atomic carrier of electricity, and no great strain on one's imagination is needed to see the reasonableness of the very close relation between the electrical properties and the chemical make-up of a solution.

Conductance (or more directly its reciprocal, resistance) of a solution is usually and most conveniently measured by means of a Wheatstone bridge of suitable type, employing alternating current to avoid polarization effects. However, the Wheatstone bridge is not the only device suitable for the purpose-several other methods well known in physics can, with suitable modifications, be used. It is beyond the limits of this discussion to go into details concerning conductance measurements. My object is to give a presentation of some of the practical aspects of the potential difference measurements, particularly applied to that potential difference which is a function of the concentration of the hydrogen ion. It should be said at this point that potential difference cannot be measured with a Wheatstone bridge, which is purely a resistance-measuring device.

1 Presented before the Chicago Section of the American Chemical Society, October 21, 1921; before the South Bend and Mishawaka Section, November 22, 1921; and before the Indiara Section, December 9 , 1921.

2 Manager of Development and Manufacturing.
When a compound is dissolved in water, some of its molecules are constantly splitting apart with electrical charges of opposite signs on the two parts. These oppositely charged ions are constantly seeking to recombine and many of them do recombine. When equilibrium is established, both the splitting-up process and the process of recombination take place at a constant rate, and the average number of free ions as compared with the total number of molecules gives us a measure of the so-called dissociation of the compound.

It is well known that the water molecule splits up into $\mathrm{H}$ and $\mathrm{OH}$ ions, but at equilibrium in pure water the total ionization is extremely small, the amount of $\mathrm{H}$ or $\mathrm{OH}$ ions at room temperature being only $1 / 10,000,000$ gram-molecule per liter of water; the water is therefore $1 / 10,000,000$ normal acid or alkaline. A neutral salt dissolved in water has no effect upon this equilibrium condition. Should an acid or base be put into the water, however, the former increases the number of $\mathrm{H}$ ions and the latter the number of $\mathrm{OH}$ ions, with the result that the rate of combination of oppositely charged ions becomes greater, and when equilibrium is established, the concentration ${ }^{3}$ of $\mathrm{H}$ and $\mathrm{OH}$ ions, respectively, will be much greater than $1 / 10,000,000$ normal.4 With an acid like $\mathrm{HCl}$, for example, it is easily possible to have such a high degree of ionization that in a molar solution of $\mathrm{HCl}$, more than 80 per cent of the hydrogen is ionized. Such an acid, in which a relatively large proportion of the $\mathrm{H}$ atoms are ionized, is regarded as a strong acid. The strength of an acid is thus related to the number of $\mathrm{H}$ ions present in the solution. If, for example, a molar solution of acetic acid be made up, only 4.3 parts per thousand of the available hydrogen will be ionized. Bases behave similarly with respect to $\mathrm{OH}$-ion concentration. These considerations bring out the distinction between available and actual hydrogen- or hydroxyl-ion concentration. In both $\mathrm{HCl}$ and $\mathrm{CH}_{3} \mathrm{COOH}$, the molar solution contains available hydrogen to the extent of $1.008 \mathrm{~g}$. per liter; but in the first instance there is present in ionized form, ready to react, about $0.83 \mathrm{~g}$., while in the second there are only $4.3 \mathrm{mg}$. Measurements of $\mathrm{H}$-ion concentration involve a direct determination of the concentration of actual ionized hydrogen present. This cannot be found by titration with standard neutralizing solutions, which gives a measure merely of total, not actual reaction.

3 In this connection the writer takes the liberty of quoting from a statement he made several years ago in L. \& N, Catalog No. 75, footnote, p. 6 : "The discrepancies observed, especially in strong acids, between the ionic concentrations as measured by conductivity methods on the one hand and with the hydrogen electrode on the other, suggest that the quantity which we call $\mathrm{H}$-ion concentration may not actually represent the degree of normality of hydrogen ions in the solution under test. Some have preferred to call this quantity 'activity.' Whatever name we choose to apply to it, the fact remains that in connection with a solution containing hydrogen ions there is a definite quantity susceptible to measurement, to the different valLes of which there correspond very definite characteristics of the solution. In this discussion we prefer to use the term 'hydrogen-ion concentration,' and in so doing we shall feel no concern regarding the objections which have been made to the use of the term."

4 This fraction is more simply expressed as $1 \times 10^{-7}$, the negative exponent of 10 signifying that the power of 10 indicated is the denominator of the fraction. It is evident that any value between $1 / 10,000,000$ and $1 / 1,000$,000 , the latter being the same as $10 / 10,000,000$, can be expressed by a factor between 1 and 10 , times $10^{-r}$. The factor need not be purely integral, $i, e_{\text {, }}$ it may be an integer plus a fraction, e. g., $6.43 \times 10^{-i}$. The same.statements hold for any decimal interval. This is the common method of expressing $\mathrm{H}$-ion concentrations in terms of normal. 
It is important to observe that, whatever the $\mathrm{H}$-ion concentration may be when a substance is dissolved in water, the product of the concentration of $\mathrm{H}$ and $\mathrm{OH}$ ions is $10^{-14}$. Hence, in designating acidity or alkalinity, it is merely necessary to mention the value of $\mathrm{H}$-ion concentration, for its $\mathrm{OH}-\mathrm{ion}$ concentration follows directly from this figure as

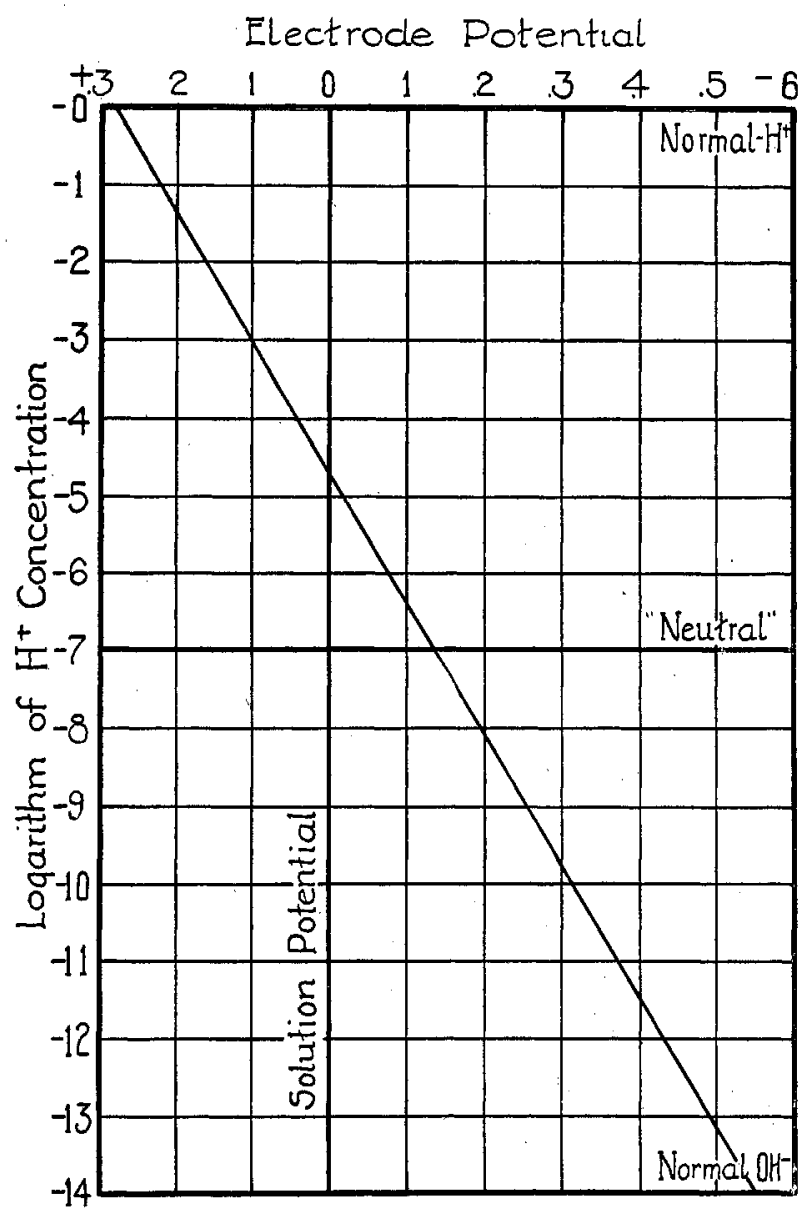

Fto. 1-Electrode potential of hydrogen electrode with reference to solution in which it is immersed, plotted against logarithm of hydrogen-ion concentration.

shown a moment ago. Another point worth remembering is that, when the $\mathrm{H}$-ion concentration is $10^{-14}$, the $\mathrm{OH}-\mathrm{ion}$ concentration is $10^{\circ}$, or normal.

If we put an electrode of any metal or of hydrogen into a solution containing the corresponding ions, there will be set up, as a result of establishment of equilibrium between the solution pressure of the electrode substance and the osmotic pressure of the ions, a potential difference between the electrode and the solution. You will recall that potential difference (measured in volts or millivolts), sometimes called voltage, bears to electricity exactly the same relation that pressure bears to water. The higher the water pressure, the greater will be the speed with which a given quantity of water passes any point in a pipe or the greater the current. Similarly, the greater the potential difference, or electric pressure, the stronger the electric current resulting when a path is provided in which it may flow. But just as water pressure can exist. statically, without causing a current of water, e. g., when the pipe is stopped, so a potential difference can exist without causing a current, e. $g$., when no circuit is provided. And it is this electrical pressure or voltage which varies as the concentration of the ions varies. It may be positive or negative with reference to the solution, $i$. e., may have a deficiency or excess of electrons as compared with the solution, this depending on the solution pressure of the metal and the osmotic pressure or concentration of the ions of that metal.

The measured values of H-ion concentration, whether they be determined colorimetrically or by the methods which are here being discussed, derive their status from Nernst's equation,

$$
\mathrm{E}=\mathrm{E}_{0}+\frac{\mathrm{RT}}{n \mathrm{~F}} \log _{e} \mathrm{C}^{+}
$$

in which $\mathrm{E}$ is the potential difference between an electrode and the solution containing the corresponding ions; $\mathrm{E}_{0}$ is the electrolytic potential, or the particular value of $\mathrm{E}$ when the ionic concentration $\mathrm{C}^{+}$in the solution is normal; $\mathrm{R}$ is the gas constant; $T$, the absolute temperature; $n$, the valence of the ion; $F$, the faraday, or 96,500 coulombs; and $\log _{\mathrm{e}} \mathrm{C}^{+}$is the natural logarithm of the ionic concentration.

Taking the hydrogen electrode specifically, this is nothing more than a coating of platinum black deposited on an inactive metal, and exposed sufficiently long to a hydrogen atmosphere to become saturated. Such an electrode is positive to the solution when the concentration of hydrogen ions is normal; zero, when the concentration is somewhat less than $1 / 100,000$; and is negative for lower values of concentration. This is shown in Fig. 1, which is a graph of Nernst's equation as applied to the hydrogen electrode. The values of potential used are based on observations, recorded in chemical literature, made with the dropping electrode or upon the curvature of the mercury surface in the tube of a capillary electrometer. They are approximately correct, probably within 10 millivolts. These potential values are plotted as abscissas, and the common logarithm of the concentration as ordinates. We have here adopted the convention of plotting the curve in the fourth quadrant, so that decreasing actual acidity is represented by a downward slope of the graph. This is also consistent with the fact that the logarithms are negative, since $\mathrm{C}^{+}$for hydrogen is always expressed as a fraction of normal, and the logarithm of a fraction is a negative number. On this scale of ordinates, -1 means $0.1 \mathrm{~N}$; -2 means $0.01 N$, etc., since $\log 1 / 10=-1$ and $\log 1 / 100=$ -2. This scale, taken positively, is called the $\mathrm{pH}$ scale, ${ }^{5}$ according to Sörenson's suggestion. Because of its convenience, biochemists have unanimously adopted the $\mathrm{pH}$ scale.

To convert a $\mathrm{pH}$ number ${ }^{6}$ to actual $\mathrm{H}$-ion concentration it is necessary only to find the number corresponding to the

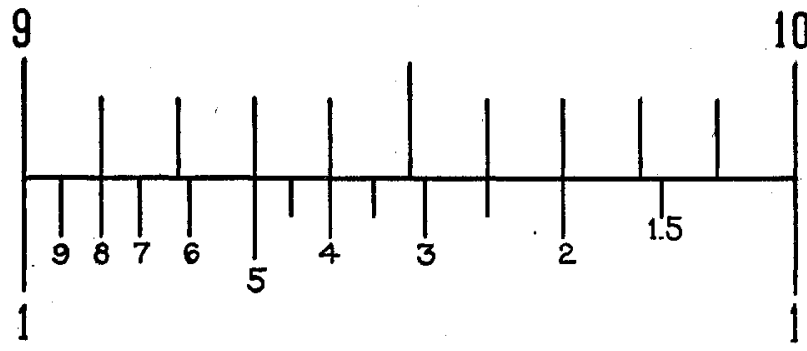

Frg. 2-Showing use of slide rule for converting $\mathrm{pH}$ into $\mathrm{C}^{+}$and vice versa. Upper scale represents scale of equal parts (whole length of rule) and lower scale the inverted logarithmic scale.

- Properly written, this should be $\mathrm{pH}_{\mathrm{H}}$, the derivation being that the number is proportional to the potential of the $H$ electrode. For convenience in typographical work, $\mathrm{pH}$ is quite generally used.

- It is unfortunate that a single method for designating acidity and alkalinity has not been adopted. There are arguments for both the $\mathrm{pH}$ system and the numerical designation of $\mathrm{H}$-ion concentration. The former, which is not as commonly used among chemists as the latter, has the advantage that $\mathrm{pH}$ values are linearly proportional to voltage, and the worker soon associates the $\mathrm{pH}$ value with perfectly definite characteristics of a solution. The latter, though it has definite physical meaning and is therefore more logical to use, is somewhat cumbersome, and greater effort is required to become fluent in its use. 
$\mathrm{pH}$ value taken as a negative logarithm. As an illustration of this conversion process, suppose we wish to determine the H-ion concentration of a solution whose $\mathrm{pH}$ number is 9.54 . The logarithm of the concentration sought is -9.54 . The negative sign means that the corresponding value is a fraction, the denominator of which is the number whose logarithm is 9.54 , or the fraction of normal concentration is $1 / 3,500$,000,000 . This mode of expression is awkward. A better way is to write it in accordance with the plan mentioned in Footnote 3 , or $\frac{1}{0.35} \times 10^{-10}$, which is $2.88 \times 10^{-10}$.

Conversion from $\mathrm{pH}$ to $\mathrm{C}$ and vice versa is easily made with a slide rule having a scale of reciprocals, or with an ordinary slide rule, by inverting the slide. The scale of equal parts then reads the portion of the $\mathrm{pH}$ number after the decimal point, while the inverted scale reads the concentration factor which is multiplied by 10 with a negative exponent greater by 1 than the characteristic (preceding the decimal point) of the $\mathrm{pH}$ number. Fig. 2 will serve as a guide in following this example on a slide rule.

We now have the following picture: An electrode of platinum black, saturated with hydrogen, in a solution; a potential difference between the electrode and the solution, depending upon the H-ion concentration. The problem is to measure this potential difference and its variations as the concentration of $\mathrm{H}$ ions changes. To make the measurement with an electrical measuring instrument requires that means be found to connect the instrument to the electrode and solution, respectively, without introducing any additional potential differences into the circuit which, in the measurement, could not be differentiated from the potential difference we are trying to measure. We could make a metallic connection to the hydrogen electrodes, which would result in no difficulty; but how shall we connect to the solution and not run into trouble? Obviously we cannot use a metal electrode, dipping it into the solution, for this would be the seat of an unknown variable electromotive force. But a liquid junction can be established at which the e.m. $\mathrm{f}$. is either negligible or very small and determinable; and if we can hold the second liquid invariable, and immerse in it a suitable electrode connected to our apparatus, the answer might be found. Although this would almost surely introduce an e.m.f. in series with the significant e. m.f., the former would be constant and determinable.

An obvious thought is to use as the second liquid, through which connection to the test solution is established, a solution of fixed H-ion concentration, in combination with a second hydrogen electrode. This gives us a connection such that the over-all potential difference will be measurable, and in which any variation of potential is due only to the changing $\mathrm{H}$-ion concentration in the test solution. Fig. 3 illustrates the idea.

The same arrangement of electrodes, with the addition of a galvanometer and tapping keys with protective resistance, as shown in Fig. 4, serves a very useful purpose enabling the user to adjust the $\mathrm{pH}$ of a solution to be very closely the same as that of another reference or standard solution. Consider A and F immersed in solutions of different $\mathrm{pH}$ values, assuming $\mathrm{B}$ to be more acid than $\mathrm{D}$. The electrical pressure (potential difference) is such that $A$ tends to cause a flow into $B$; at the same time $F$ tends to cause a flow of electricity into D, but the pressure is not so great, since $\mathrm{D}$ is less acid. If the circuit be elosed through the galvanometer, a current flows, and causes a deflection, because of these unbalanced tendencies. Now let us imagine

\footnotetext{
The arrangement and the application here described were suggested by the writer in Science, N. S., 62 (1920), 18, where a more complete description may be found.
}

that $B$ is a solution of exactly the proper acidity, and that we wish to render $D$ as nearly like $B$ as possible. All we need to do is to add $\mathrm{H}$ ions to $\mathrm{D}$ until the galvanometer shows no deflection when the key is closed. This method requires no

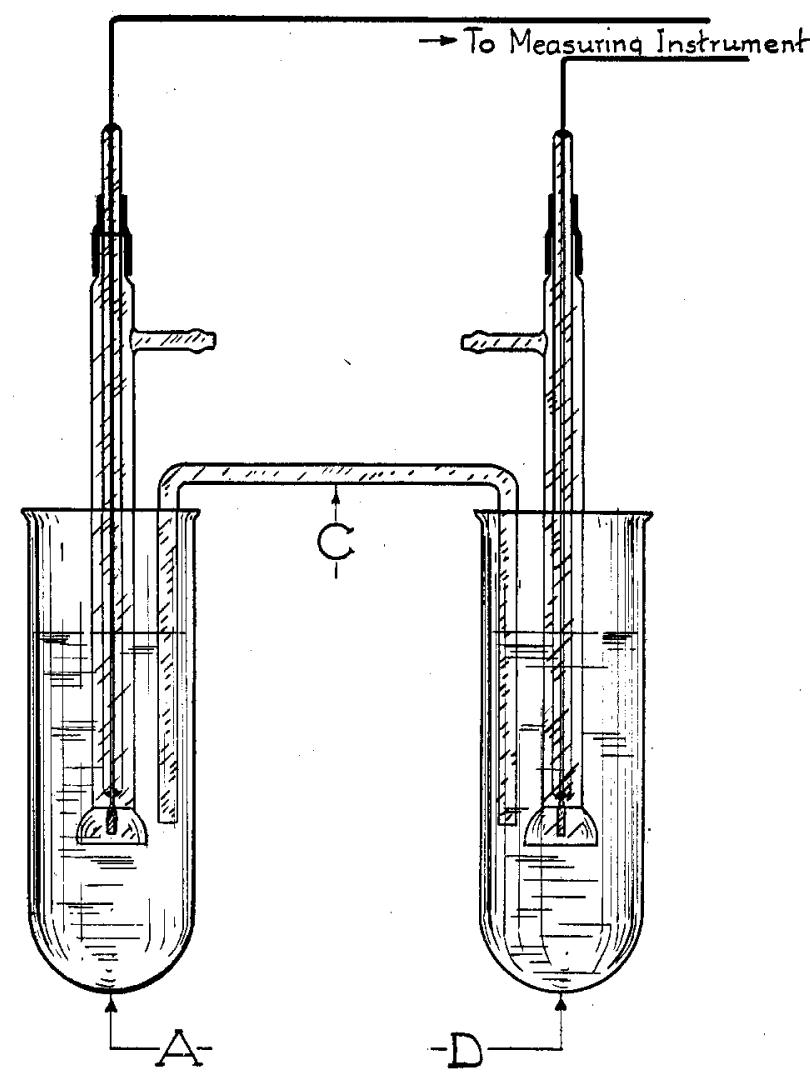

Fig. 3-A possible "gas-chain" arrangement, in which $A$ is the standard solution, $\mathrm{D}$ the test solution and $\mathrm{C}$ the connecting tube. The latter is filled with a neutral conducting liquid, like $\mathrm{KCl}$; the ends are plugged. with cotton wool to prevent diffusion. The hydrogen electrode potential in $A$ remains fixed, while that in $D$ varies with the acidity of $D$.

knowledge of units-only of manipulation. An illustration would be to use a salt solution in $B$, and to titrate the corresponding acid with the corresponding base in $\mathrm{D}$. Or, we might put a solution of known $\mathrm{H}$-ion concentration in $\mathrm{B}$, and use the arrangement in lieu of the more usual color indicator. This method has found much application in the adjustment of bacteriological culture media. Each end-point, of course, requires a particular solution of known $\mathrm{pH}$ value. By this and the other electrometric methods to be described, it is easily possible to titrate to within $0.01 \mathrm{pH}$ unit. This is far closer than we can work with the best color indicators under the most favorable conditions.

The simplicity of application of color indicators makes them a very useful tool in the chemist's kit. As to the limits of their usefulness, we might say in general that turbidity or color in solutions, poor lighting conditions, and some kinds of salts and proteins make accurate determinations difficult, and when such conditions are encountered, the chemist should be on his guard lest he assume that he is getting greater accuracy than is actually the case. With a clear and colorless solution serving as the vehicle for a simple, well-defined reaction, we can expect an absolute accuracy of $=0.3 \mathrm{pH}$ unit or slightly better. For many purposes this is ample, since, at the equivalent point of a titration, a few drops suffice to change the concentration of the $\mathrm{H}$ and $\mathrm{OH}$ ions by several $\mathrm{pH}$ units. The result is that in a simple titration with strongly ionized reagents almost any one of the usual indicators, such as methyl orange, phenolphthalein, 
azolitmin, and many others investigated by different workers, give a sufficiently accurate indication of the neutral point.

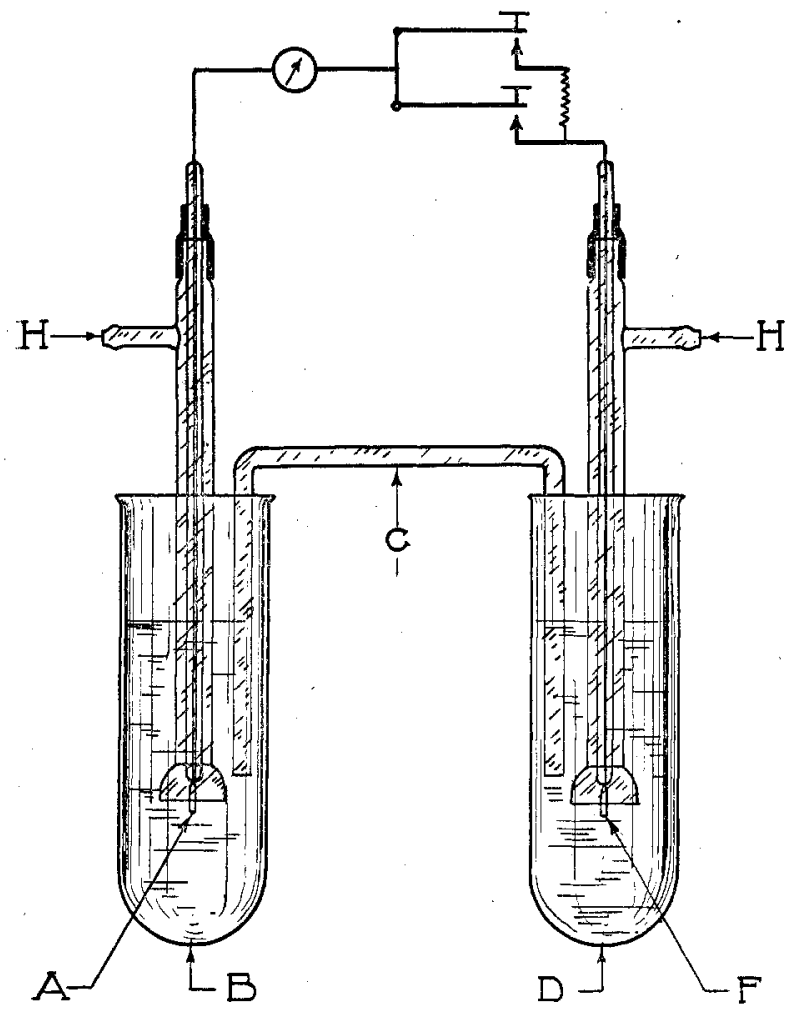

Fro. 4-Showing the application of the gas chain shown in Fig. 3 to simple titrations to a desired end-point. The hydrogen electrodes are connected through a galvanometer, to show equality of potential, and. suitable tapping keys.

If, however, we desire to adjust an acidity or alkalinity in a particular region where there is no sudden change, it is necessary to be careful in the selection of the indicator, which should change color rapidly in the region in which we are interested. Methyl red, for example, could not be used as an indicator outside the limits 4.5 to 6 . Another important point to observe is that all indicator ranges have been established with the hydrogen electrode, and that the color indicator is a secondary rather than a primary method in analysis or control. We may make the summary that the electrometric method is superior to the colorimetric method in the fact that with it we may often secure information that would be impossible to obtain by the indicator methods; and that in any case to which the hydrogen electrode can be applied, the results will be much more accurate than with an indicator. Where the color indicator methods can be used, they are to be recommended for their convenience.

Both the colorimetric and electrometric methods can to good advantage employ solutions of known $\mathrm{pH}$, for check tests if for no other reason. Sörenson, Clark and Lubs, and others have worked out formulas for so-called "buffer mixtures" of any desired $\mathrm{pH}$ value. The "buffer mixture" is a mixture, such as an acetate, phthalate, or phosphate, which has great stability as regards changes in $\mathrm{pH}$. For example, although very careful experiments show pure water to have an $\mathrm{H}$-ion concentration of $10^{-7}$, measurement on distilled water of high purity will seldom yield this value, because even the slightest acid or alkaline contamination (e. g., carbon dioxide from the air) may change this exceedingly small concentration tenfold or a hundredfold. We can, however, produce a stable solution having a concentra-

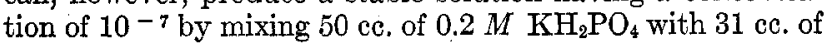
the same strength $\mathrm{NaOH}$, and diluting to $200 \mathrm{cc}$. Specific application of such a solution has already been indicated in the comparison method of titration, employing only a galvanometer.

Returning from this brief digression to the question of how to find quantitatively the variation in potential of the hydrogen electrode with reference to the test solution, we have seen that an electrode of fixed and determinable potential in an unvarying solution can be added to the chain, and that it affords a means for access to the quantity to be measured. Any such auxiliary electrode will do; but the type which has come into most general use, and which is generally accepted as the most satisfactory and convenient is the so-called calomel half-cell, or "calomel electrode." It has a variety of modifications in design and execution. Essentially it consists of pure mercury as the electrode proper, covered with a layer of pure calomel in solution of potassium chloride of known saturation, which itself has been saturated with calomel. The cell with $0.1 N \mathrm{KCl}$ has probably been most closely studied; but there are certain advantages in the use of a more concentrated potassium chloride solution, which will be pointed out later. The cell has a side arm, or siphon tube, through which connection is established with the solution under test. Quite commonly, where accurate measurements are desired, a "connecting vessel" is used, containing saturated potassium chloride solution. This is interposed between the test solution and the calomel electrode for the purpose of eliminating liquid contact potentials. Since the latter seldom exceed a few millivolts, the connecting vessel can, in most practical work, be omitted. The common arrangement, known as a "gas chain," is shown in Fig. 5.

With an arrangement of this kind, we know that a certain potential difference exists between the mercury and the hydrogen electrodes, and that to any particular potential difference there corresponds one particular value of $\mathrm{H}$-ion concentration. If we assume for the moment that this potential difference can be measured, we can apply our working formulas,

$$
\begin{array}{r}
\mathrm{V}=v-0.0591 \log \mathrm{C}^{+}, \\
\text {or, since } \mathrm{pH}=-\log \mathrm{C}^{+}, \\
\mathrm{V}=v+0.0591 \mathrm{pH},
\end{array}
$$

to the determination of either $\mathrm{C}^{+}$or $\mathrm{pH}$. The formulas come directly from Nernst's equation, which has previously been explained. In them $\mathrm{V}$ is the measured voltage, and $v$ is the

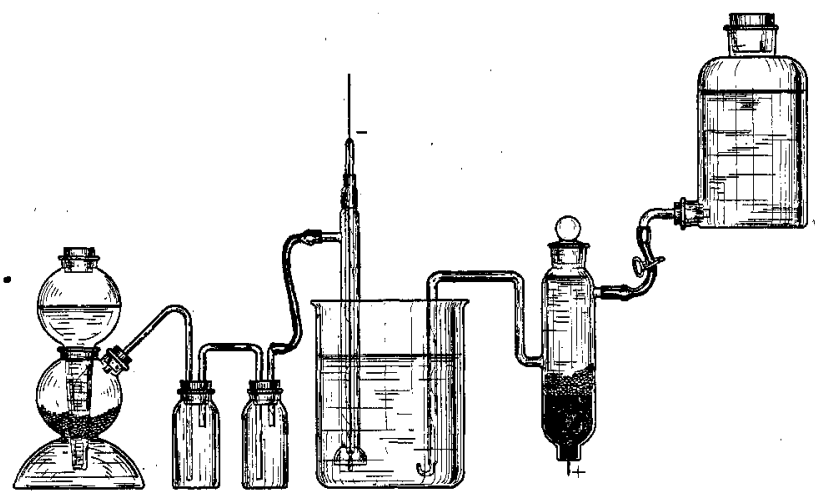

Fig. 5-Sketch of commonly used gas chain, showing hydrogen electrode supplied with hydrogen from Kipp generator, purified in wash bottles; calomel electrode with rubber tube connection to stock solution of $\mathrm{KCl}$. Both electrodes in electrical contact with solution under test in beaker.

voltage which would be measured if the hydrogen electrode were in a solution having normal concentration of hydrogen ions; for in such a solution $\mathrm{C}^{+}=1, \log 1=0$; hence $\mathrm{pH}=0$. Fig. 6 clearly illustrates the formulas. In this figure length 
of line is proportional to voltage; AD represents the measured voltage, and $A B$ the voltage which would exist between the calomel electrode and a hydrogen electrode in a solution of normal $\mathrm{H}$-ion concentration. AO represents what is known as the electrode potential of the calomel electrode $(0.560$ volt at $18^{\circ} \mathrm{C}$. for a $1.0 \mathrm{~N} \mathrm{KCl}$ solution) although it is to be noted that this does not enter into our computations. The

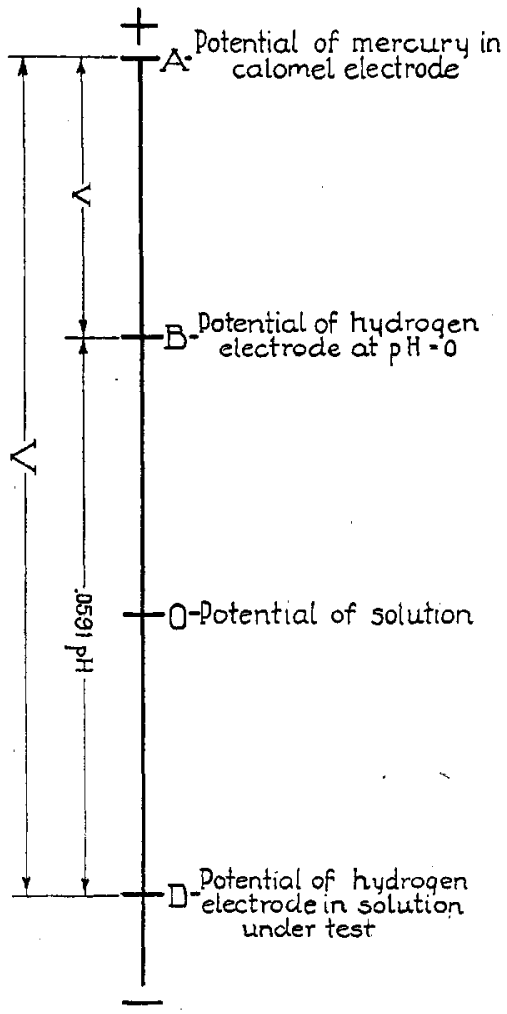

FIG. 6--Diagrammatic representation of relative potentials of calomel and hydrogen electrodes, and solution potential. This gives a graphic illustration of the working formula for the type of gas chain shown in Fig 5 .

rent from the hydrogen electrode, for to do this do this would change its potential. We may compare it with a standpipe of small diameter, the level of the water in which changes if ever so little water be drawn off. Our problem in measurement of this voltage between hydrogen and calomel electrodes-in other words, of the gas chain - then resolves into this: It must be measured without permitting any appreciable flow of electricity through the electrodes.

In physics we have three methods, which have long been well known, for accomplishing this purpose. These are, first, the electrometer, of the quadrant type, which is relatively unimportant in this discussion; second, and also of small importance, the charging of a condenser by connection to the gas chain, and measuring the charge with a so-called ballistic galvanometer; the third and really important method is that employing a means for balancing a measurable, adjustable, potential difference against the unknown. To return to the analogy which has been used several times in this presentation, it may be likened to some such scheme of measuring the pressure of water in a standpipe by using a second standpipe in which the head, and hence the pressure, is adjustable and measurable, and "bucking" the second against the first. If, on opening the valve connecting the two pipes, there is no flow in either direction, as shown by a flowmeter, the two pressures are equal, and the first is known, since it is equal to the second which is measurable.

\section{* Potentiometer Methods}

The plan of connections underlying all potentiometer or compensation methods is shown in Fig. 7. The similarity with this of the connections used in what is known as Hildebrand's method (Fig. 8) is apparent. The difference between the usual potentiometer method and this is that in the former a standard cell is used to establish a known fall of potential along the measuring circuit, while in the latter the fall of potential is ascertained at any one setting by. reading a voltmeter. We ohserve that in each instance a source of current is needed to produce the necessary potential difference to balance that of the gas chain, and a galvanometer is required for indicating when that balance has been established.

Within the past few years a number of instruments have appeared on the market; or have been described in the journals, which employ the potentiometer principle. Among them may be mentioned the "ionometer," of Bartell, which followed McClendon's description of a direct reading potentiometer; another instrument called an "ion-o-meter," described by Elliott; the Bovie potentiometer; the pyrovolter, employing the balanced potential principle; the Wendt titration outfit, employing Hildebrand's connections; the Kelley electrometric titration apparatus, designed for determinations of chromium vanadium and manganese in alloy steels, and utilizing the change in potential during an oxidation-reduction process; the Roberts outfit, used in determining minute quantities of ferrous in the presence of ferric oxides; the Eppley titration apparatus; Hendrixon's improvised potentiometer, for iodometric titrations; the Type $\mathrm{K}$ potentiometer, the portable $\mathrm{H}$-ion potentiometer, and the automatic recording potentiometer of Leeds \& Northrup Co.; and a number of others. With a list as formidable as this before him, the poor chemist, lacking guidance, must be quite at sea in the matter of making"the proper selection for his particular problem.

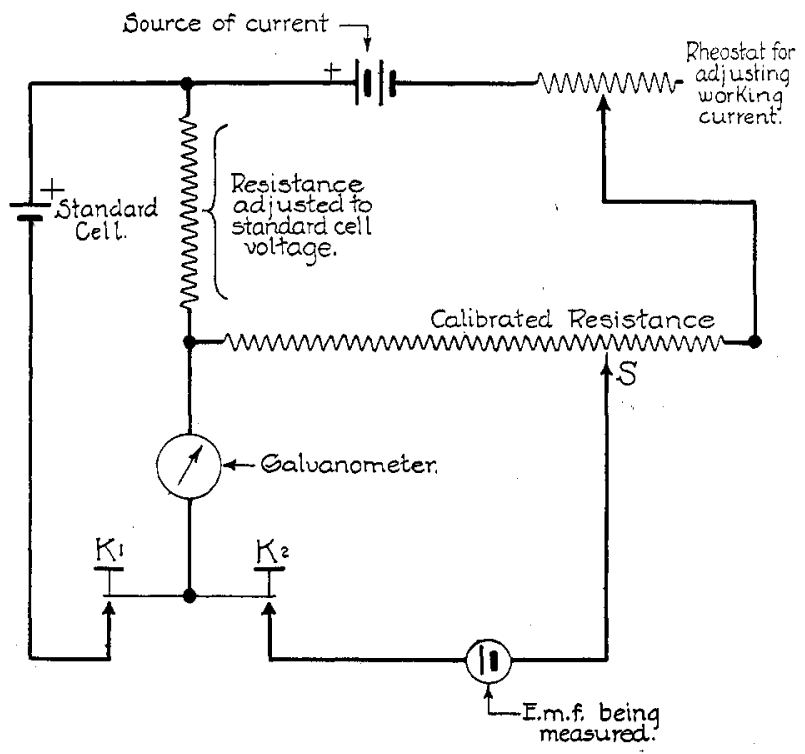

Fig. 7-Potentiometer circuits. To operate, $K_{1}$ is tapped and theostat in upper circuit adjusted for zero deflaction of galvanometer. This establishes a known current in the calibrated resistance. For meas. uring unknown e, $m$. $f_{\text {., }} K_{2}$ is tapped and $S$ moved until galvanometer shows zero. The position of $S$ is then read on a scale which is calibrated directly in volts. 


\section{Selection of Apparatus}

It is impossible, of course, to discuss each of these instruments, and state where it is applicable. The writer can give only a few general suggestions which will be' helpful in determining what outfit best suits certain requirements.

Is THE HYDROGEN ELECTRODE APPLICABLE?-In the first place, we must know whether or not the hydrogen electrode will be well behaved in the solution under test. There are many complex solutions, containing substances that are likely to "poison" the electrode and cause it to disregard with great obstinacy the expectation that it will observe Nernst's law. The best course is either to borrow an outfit from someone, or to let someone who has an outfit test the solution in which you are interested. Some work has been done on "poisoning" of electrodes, and the writer has been informed that a method has been devised for restoring "poisoned" electrodes, and removing from them the tendency to misbehave at any future time. Some of the "poisons" that have been mentioned are formaldehyde, sulfides, ammonia, and certain nitrates. There may be other substances in the solution upon which the platinum black in the electrode exerts a catalytic action. In solutions containing charged colloidal particles, it is impossible to measure the H-ion concentration because of the presence of these particles. According to Bovie, the silver remaining in a solution after precipitation with hydrochloric acid is sufficient in quantity to "poison" the electrode completely.

It is well to have at hand a buffer solution of known pH value which is altogether free from suspicion of poisoning the electrode. An electrode which in the test solution behaves peculiarly can then be quickly tested in the buffer solution, to determine whether or not it is poisoned. Should it, in this solution, give an indicated voltage far different from the value it should give for the known $\mathrm{pH}$, a new electrode should be used. Several tests, on different electrodes, should be made before the hydrogen electrode is rejected as a possibility.

WHAT IS THE DESIRED ACCURACY OF MEASUREMENT?-Assuming that the hydrogen electrode will work, we should decide as to the accuracy with which the measurements are to be made. The impression prevails that instruments of highest accuracy and sensitivity are requisite to any kind of $\mathrm{H}$-ion measurements. In ordinary works and laboratory processes high sensitivity is a hindrance rather than a help, because of the likelihood of the operator to draw erroneous conclusions from indications that are caused by insignificant factors. In general, solutions, and conditions of temperature and pressure, are not reproducible within very narrow limits, and other unknown and uncontrolled sources of small error are always likely to be present. For all practical purposes, an absolute accuracy of from 5 to 10 millivolts in the measuring instrument, and a sensitivity in the galvanometer of 2 to 5 millivolts under conditions of test, $i$. e., in the gaschain circuit, is ample. This accuracy corresponds to $0.1 \mathrm{pH}$ unit, which is far closer than can be measured with a color indicator under the most favorable conditions.

For investigations involving the determination of another decimal place in a physicochemical constant, or the test of a theoretical equation, it is obvious that none but the highest grade potentiometer should be used; in conjunction with a highly sensitive galvanometer. It is obvious also that the use of such instruments imposes an obligation to exercise the greatest possible care in controlling all the conditions of the experiment. The conditions, and their control, lie outside the limitations upon this discussion.

Once the chemist has decided how accurately he wishes to measure, his choice of apparatus among the different available kinds will be largely influenced by his own ideas as to suita- bility of design and convenience of manipulation of the apparatus under consideration, and, to a minor degree, upon the persuasive power of the salesman offering it.

\section{The Calomel Electrode}

There is little choice among the various available designs. Each has some merits. Simplicity in this as well as in other parts of the apparatus is much to be desired. As regards the selection of degree of saturation of potassium chloride solution, concentrations greater than $0.1 \mathrm{~N}$ are to be recommended. Mudge and Fales have shown that the saturated potassium chloride electrode is extremely stable, that it minimizes resistance of the gas chain, and that slight changes in saturation

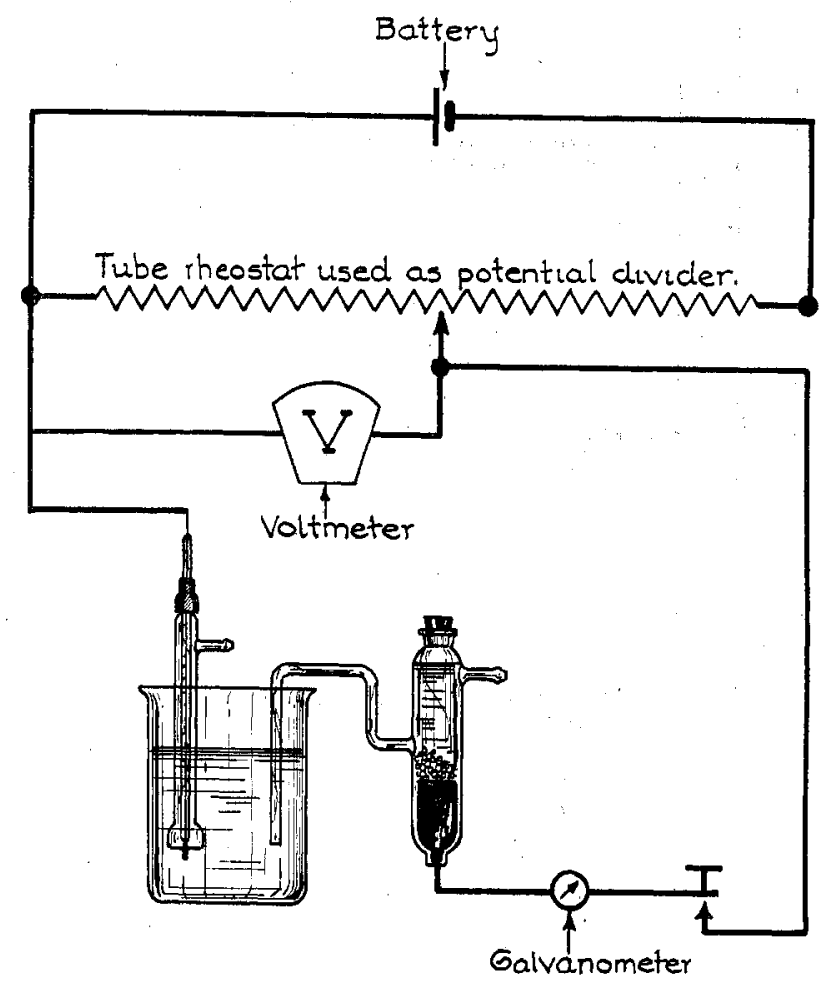

FIg. 8-Hildebrand's connections for measuring gas-chain voltage. The e. m. f. from the gas chain is balanced by adjustment of the potential divider until galvanometer shows zero. The voltmeter, permanently connected as shown, then reads the potential difference which was recuired to balance the unirnown e. m. f.

have very little effect upon its potential. The same statement holds for the $N \mathrm{KCl}$ solution to a lesser degree. The latter has an advantage over the former in that no troublesome "creeping" of solution and crystallization of salt outside the vessel occurs, although in the former case the trouble from creeping can be obviated by setting limits to it with vaseline or paraffin.

The reservoir vessel should contain potassium chloride of the same concentration as that in the cell, saturated with calomel. The cell should be flushed out from time to time by allowing some of the stock solution to run through the siphon arm. This prevents contamination of the cell from the testing solution. In flushing, care should be taken to displace all air bubbles in the siphon tube, since these introduce a high resistance into the galvanometer circuit and render the indications sluggish and insensitive.

In work of the degree of accuracy we are considering, the connecting vessel may be omitted for reasons already stated. Errors due to liquid junction potentials are least when a calomel electrode with saturated potassium chloride is used. 


\section{The Hydrogen Electrode}

Successful deposition of platinum black on the electrode is a fairly simple matter, for which there are many methods. A procedure which the writer has found invariably successful is to use a weak solution of platinic chloride-about 2 per cent -and, after having most thoroughly cleaned the platinum, introduce the electrode and a piece of clean platinum wire, or another electrode, into the solution. These two electrodes are connected to two dry cells in series. As soon as there is a copious evolution of gas, reverse the connections, and continue reversing at the same indication, for several minutes. The intervals between reversals will be 10 to 15 seconds.

Some workers prefer to use gold as the base upon which to deposit the platinum black, while others have gold-plated their platinum base. Presumably this shortens the time for the electrode to reach equilibrium because of the smaller absorption of hydrogen by gold than by platinum.

The basis for choice of any one of the many forms of hydrogen electrode described in the literature cannot be gone into here. In general, the bubbling type (Hildebrand, Bovie, Wendt) is satisfactory for a great variety of measurements. ${ }^{8}$

\section{SOURCE OF HYDROGEN}

The most convenient source of hydrogen is the steel drum containing the compressed gas, readily obtainable in most cities. Electrolytic hydrogen is desirable. The drum should have a reducing valve for regulation of gas pressure, and the gas is led to the apparatus preferably through copper tubing or undercured gum tubing, to reduce loss of gas by diffusion. Electrolytic generators on a laboratory scale have been used with success, employing sodium hydroxide with nickel or iron electrodes. The Kipp generator is also a suitable source. In any case, the gas should be passed through wash bottles containing solutions for removal of such impurities as oxygen, arsine (from impure zinc in Kipp generator), acids, and possible traces of foreign gases which might previously have been in the hydrogen cylinder. A common practice is to pass the gas first through alkaline permanganate, then pyrogallol, and finally pure water. When small quantities of solutions are being titrated or tested, e.g., in a culture tube or other small vessel, the gas itself serves admirably as an agitator. In larger vessels, a mechanical stirring device is necessary for intimate mixing during a titration.

\section{Test of Apparatus}

One test has already been mentioned, to determine the condition of the hydrogen electrode. The same kind of test is excellent as an over-all check of the apparatus. This consists of using a standardized buffer solution of any convenient $\mathrm{pH}$ value in place of the test solution, and measuring the voltage. If this is only several (10 to 20$)$ millivolts different from the calculated value for that particular $\mathrm{pH}$, it is probable that the difference is a departure in the calomel electrode from the assigned value for the concentration of potassium chloride being used, and to other sources of minor e.m.f.'s. Adjustment can then be made in the calculations. A check on this test is to use another solution of different $\mathrm{pH}$ and to note whether the same difference in millivolts is observed.

It is to be noted that with a preliminary test of this sort it is unnecessary to devote much painstaking work to making up the calomel electrode. The only requirement is that its electrode potential be unvarying, and this condition becomes established within several days after preparation of the cell.

- Other practical points in connection with the use of the hydrogen electrode are mentioned in Leach's "Food Inspection and Analysis," 4th edition, 1027.
MEASUREMENT OF $\mathrm{PH}-$ When it is desired to know the actual $\mathrm{H}$-ion concentration or $\mathrm{pH}$ of a given solution, a direct measurement of voltage of the gas chain is sufficient, from which the desired value is calculated.

\section{Conversion of Measured Voltage into pH}

This is accomplished by using the formula previously given: $\mathrm{pH}=\frac{\mathrm{V}-v}{0.0591} . \quad \mathrm{V}$ is the observed voltage, and $v$ the calomel cell potential referred to the hydrogen electrode at $\mathrm{pH}=0$; the factor 0.0591 is the number of volts corresponding to one $\mathrm{pH}$ interval at $25^{\circ} \mathrm{C}$. There is a small temperature correction which in routine work at "room" temperature may be disregarded. The otherwise necessary computations are eliminated by the use of tables. ${ }^{9}$ A still simpler and more convenient way is to use a specially designed slide rule of straight or circular pattern, as recently described by the. writer. ${ }^{10}$ An ordinary slide rule is also a great convenience, although in this case a mental subtraction $(\mathrm{V}-v)$ must first be made.

\section{Electrometric Titration}

This application of the hydrogen electrode with its measuring and indicating instruments is one of the most important, from the chemist's standpoint. Titration with a standardized acid or basic solution to some particular end-point (equivalent point), and adjustment of a medium to a particular $\mathrm{pH}$ value for optimum experimental conditions, are illustrations.

The usual titration curves, of the type obtained by Hildebrand, ${ }^{11}$ are obtained by plotting voltage or $\mathrm{pH}$ against volume of solution added. Near an equivalent point there is a sudden and usually large change in voltage. This change, shown by a sudden large galvanometer deflection, may be taken as an indication of the equivalent point, or where higher accuracy is needed, a curve may be plotted and the equivalent volume more closely estimated. An accuracy of one or two parts per thousand is easily obtained. When the $\mathrm{pH}$ of an equivalent point is known, or when it is desired to adjust a solution to a definite $\mathrm{pH}$ value, the voltage may be set for the proper value, and titration continued until the galvanometer shows a balance, In all such measurements a light tap on the galvanometer key should be employed until a balance has been nearly obtained, after which the circuit may be kept closed. This precaution avoids upsetting equilibrium conditions in the gas chain.

In a limited time it is impossible to do justice to an enumeration, even with the briefest descriptions, of the many successful applications that have been made of the hydrogen electrode. For these reference must be made to the literature. In Clark's excellent book "The Determination of Hydrogen Ions" there is given a bibliography, classified according to applications, which gives one an excellent idea of what has been done up to 1920. Since that time other applications have been made, and more are added with each new issue of the chemical journals.

One application has been suggested by several chemists interested in teaching, the value of which is borne out in the experience of those who have made electrometric titrations with the hydrogen electrode. It deserves special emphasis. There is probably no method or apparatus that can be put in the hands of a student which is so illuminating and which so readily explains the meaning of such terms as "acidity," "actual reaction," "total reaction," "alkalinity," "hydroly-

- For example, Schmidt and Hoagland, Univ. Calif. Puh. Physiol., 8 (1919), 23.

10 Science, 54 (1921), 153.

il J. A m. Chem. Soc., 35 (1913), 847. 